\title{
Molecular Aggregation and Ultrasonic Properties of Hydroxypropyl-Cellulose Films
}

\author{
Akira TANAKA, Hiroshi ONODA, ${ }^{*}$ and Koh-hei NITTA ${ }^{* *, \dagger}$ \\ Department of Materials Science, University of Shiga Prefecture, \\ 2500 Hassaka, Hikone, Shiga 522-8533, Japan \\ ${ }^{*}$ Department of Polymer Chemistry, Kyoto University, \\ Yoshidahonmachi, Kyoto 606-8317, Japan \\ ${ }^{* *}$ School of Materials Science, \\ Japan Advanced Institute of Science and Technology, \\ 1-1 Asahidai, Tatsunokuchi, Ishikawa 923-1292, Japan
}

(Received January 12, 2000)

\begin{abstract}
Ultrasonic properties of hydroxypropyl-cellulose films showing various cholesteric colors were examined as a function of temperature. The variously cholesteric colored films was prepared by casting from water and organic solvents. Cholesteric pitch was controlled mainly by heating time. Decrease of cholesteric pitch in the colored films leads to the ultrasonic velocity increase and ultrasonic attenuation decrease. A comparison of ultrasonic properties between planner and normal direction to the film demonstrated that interactions of inter-layer are more intensive compared with those between cholesteric domains.

KEY WORDS Hydroxypropyl-Cellulose/Ultrasonic Properties/Liquid-Crystalline Polymers/Cholesteric Structure / Molecular Aggregation /
\end{abstract}

Liquid-crystalline materials exhibit optical anisotropy due to long range orientational order. The liquidcrystalline state differs from isotropic liquids not showing long range ordered structures and from crystals showing cubic long range orders. Thus, the association or aggregation of liquid-crystalline polymers is of interest for better understanding of the aggregation properties of polymers.

In the past decade, numerous studies ${ }^{1-3}$ show that most cellulose derivatives form cholesteric liquid crystalline phases in solution or in the bulk. In particular, hydroxypropyl-cellulose (designated as HPC) has been widely studied because mesophases can be easily formed in water and common organic solvents. ${ }^{4-6}$ In the case of HPC, cholesteric structures are promoted in mesophases, in which right handed helicoidal arrangement of the polymer molecules ${ }^{4,7}$ behaves as a rod-like molecule. $^{8-10}$

The cholesteric structure consists of rotating layers of rod-like molecules ${ }^{11,12}$ and is characterized by the presence of a helical twist of uniform pitch. If cholesteric materials have cholesteric pitch comparable to the wavelength of visible light, it is expected from de Vries theo$\mathrm{ry}^{13}$ that the materials will show cholesteric color due to the selective reflection of the visible light caused by the uniform cholesteric twisted structure.

Ultrasonic waves travel by molecular interactions and thus are sensitive to minute changes in the material structure. Previously, we demonstrated that the development of cholesteric structures in HPC solutions during the solvent evaporation process can be monitored using ultrasonic measurements. ${ }^{14}$ In this work, molecular aggregation in HPC films showing various cholesteric colors was investigated by ultrasonic measurement.

\footnotetext{
${ }^{\dagger}$ To whom correspondence should be addressed
}

\section{EXPERIMENTAL}

\section{Materials}

Hydroxypropyl-cellulose used in this study is a commercial polymer, HPC-SL produced by Toyo Soda Co. Ltd. The structural formula is shown in Figure 1. According to Horio et al., ${ }^{15}$ weight average molecular weight is 164300 , number average molecular weight 41600 , degree of molar substituent is 3.5 , and degree of substituent 2.3 .

\section{Preparation of Cholesteric Colored Films}

HPC films were prepared by casting from water, ethanol, acetic acid, and dioxane solutions. Most of the cast films prepared by free-standing showed no cholesteric colors. However, colored films could be obtained by the following procedures.

All samples were dissolved in these solvents ( $\mathrm{ca} .30$ $w t \%)$ for at least two weeks. The solutions was poured on a pan with flat Teflon surface, and solvent was evaporated in a normal laboratory environment. When the solution began to show iridescent color, it was wrapt up in

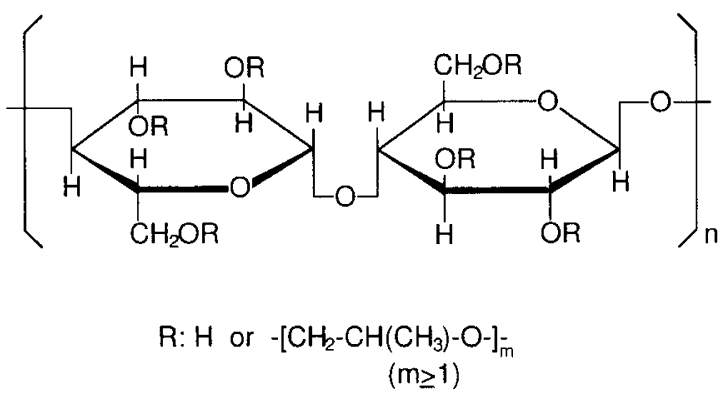

Figure 1. Structural formula of hydroxypropyl-cellulose. 
saline film to stop evaporation and allowed to achieve a high degree of order. Taking off the wrap film, the solution was evaporated slowly up to $c a .80$ to $85 \mathrm{wt} \%$ at a fixed temperature below the glass transition temperature of HPC ( $\left.\mathrm{ca} .25^{\circ} \mathrm{C}\right)^{16}$ and above the freezing point of the solvent. Finally, the colored films can be obtained by heating concentrated solution. Cholesteric color dramatically changed with annealing conditions.

The HPC-acetic acid system showed cholesteric color at higher concentrations. Colored films could be obtained only by heating the corresponding colored solution at $120^{\circ} \mathrm{C}$ for $2 \mathrm{~min}$.

Mesophase solution of HPC-ethanol was obtained by allowing ethanol to evaporate slowly in a refrigerator (at $c a .7^{\circ} \mathrm{C}$ ) up to $c a .85 \mathrm{wt} \%$. Various colored films could be prepared by annealing the concentrated solution at 120 ${ }^{\circ} \mathrm{C}$ for various times. For example, violet films could be obtained by annealing for $10 \mathrm{~min}$, green ones for $12 \mathrm{~min}$, and red ones for $15 \mathrm{~min}$. This marked variation is indicative of thermotropic liquid-crystalline characteristics. ${ }^{17-21}$ For HPC-dioxane, red, yellow, green, and violet films can be obtained in the same manner by carefully evaporating at appropriate temperature because the evaporation of dioxane is too rapid for mesophase to develop, and also because the freezing temperature of dioxane is relatively high $\left(11.8^{\circ} \mathrm{C}\right)$.

In the case of HPC-water showing iridescent color at lower critical concentrations, colored films showing relatively longer wavelength (namely, red, yellow, and green) could not be obtained. However, blue and violet films could be obtained by repetition of annealing at 120 ${ }^{\circ} \mathrm{C}$ for $10 \mathrm{~min}$.

All films prepared were dried thoroughly under vacuum for at least few weeks and kept in a dry atmosphere. For comparison, colorless HPC films were prepared from the melt at $260^{\circ} \mathrm{C}$ by compression molding.

\section{Measurements}

UV-Visible spectrophotometric measurement for HPC cast films was made at room temperature using a Jasco UVIDEC-1 Double-Beam Spectrophotometer. Film specimen was mounted between two glass slides and the intensity of transmitted light was recorded with an automatic dual beam spectrophotometer with unpolarized incident light at 310 to $760 \mathrm{~nm}$.

Ultrasonic measurements were performed by a pulse method using transducers with resonant frequency of $150 \mathrm{kHz}$. Ultrasonic velocity $v$ and attenuation coefficient $\alpha$ were measured as a function of temperature (from room temperature to $80^{\circ} \mathrm{C}$ ) for $\mathrm{HPC}$ films showing various cholesteric colors. Velocity and attenuation of ultrasonic waves in directions parallel and perpendicular to the film surface were investigated. The attenuation of ultrasonic waves in the direction perpendicular to the films was calculated by considering reflection of ultrasonic waves. The ultrasonic velocity was determined from the propagation time and distance between transducers. The dynamic mechanical properties were measured at $110 \mathrm{~Hz}$ with a Rheo-vibron Model DDV-IIc. The sample specimen was cooled from room temperature to about $-160^{\circ} \mathrm{C}$ and then heated at a rate of $c \alpha .1^{\circ} \mathrm{C} \mathrm{min}^{-1}$.

\section{RESULTS AND DISCUSSION}

Transmittance of HPC films cast from ethanol, acetic acid, and dioxane is plotted against the wavelength in Figure 2. The caption in the figure indicates the visible color observed at normal angle to the film surface. Open circle denotes normalized transmittance and closed circle net transmittance with subtracted background (solid lines). The transmittance curve of the background was considered as a Lorentz curve. This correction of background is plausible because transmittance curves of colorless films prepared by compression molding ${ }^{21}$ can be described by a Lorentz curve (see Figure 3 ).

As shown in Figures $2 a-2 c$, the wavelength of $U V$ absorption is in good agreement with that of visible color, indicating that colors of films are due to the selective reflection of visible light caused by cholesteric order.

For colored (namely, yellow, green, and violet) films cast from acetic acid, temperature dependence of ultrasonic velocities in the directions perpendicular and parallel to the films surface is shown in Figures 4 and 5, respectively. Velocity in both directions decreased monotonously with increasing temperature, reflecting "softening" of materials. Ultrasonic velocity was in the order of violet, green, and yellow over the temperature range: i.e., velocity increased with decreasing cholesteric pitch. The increase in velocity in the perpendicular direction may be due to stronger molecular interaction arising from smaller pitches, or from closer packing of cholesteric layers. The closer packing of cholesteric layers leads not only to stronger interaction between cholesteric domains but increase in the modulus of the cholesteric domains, resulting in increase in velocity in the planner direction.

Velocity in the perpendicular direction to film surface is higher than that in the parallel direction for all colored films. Velocity in the both direction of the compression-molded film was the same and its value was $2.0 \pm 0.1 \times 10^{5} \mathrm{~cm} \mathrm{~s}^{-1}$ at room temperature. This value is lower than that in the perpendicular direction of yellow film and higher than that in the planner direction of violet film. This suggests that compression-molded film is isotropic.

As demonstrated by Samuels, ${ }^{22}$ polymer molecules of HPC films align preferentially in the plane of the film, and HPC shows a planner cholesteric arrangement in the solid state. Thus, ultrasonic waves in the direction perpendicular to the film surface propagates by interlayers interaction, whereas those in the direction parallel to the film surface propagate by planner intermolecular (inter-rods) and inter-domains interactions (see Figure 6). Interactions of inter-layers and inter-rods would be stronger compared with interactions between cholesteric domains because ultrasonic velocity in the planer direction is lower than that in the perpendicular direction.

Attenuation coefficients of ultrasonic waves in the perpendicular direction are plotted against temperature for HPC films cast from acetic acid in Figure 7. Attenuation in the perpendicular direction was almost independent of cholesteric color (or pitch) and temperature. Attenuation in the planner direction depends drastically on 
(a)



(b)



(c)



Figure 2. UV-transmittance of HPC cast films from (a) ethyl alcohol, (b) acetic acid, (c) dioxane. Captions indicate visible colors at normal angle to the film surface. Open circle denotes normalized transmittance, closed circle net transmittance, and solid line background intensity given by a Lorentz function.



Figure 3. UV transmittance of colorless films prepared by compression molding. Open circle denotes normalized transmittance and the solid line the best fit to the Lorentz curve.

cholesteric pitch and on temperature (see Figure 8). Attenuation in the planner direction is almost constant at lower temperature and increases rapidly above a critical temperature. This temperature decreases as cholesteric pitch becomes longer: for yellow film it is $c a .40^{\circ} \mathrm{C}$, for green ca. $55^{\circ} \mathrm{C}$, and for violet one $c a .65^{\circ} \mathrm{C}$. Since increase

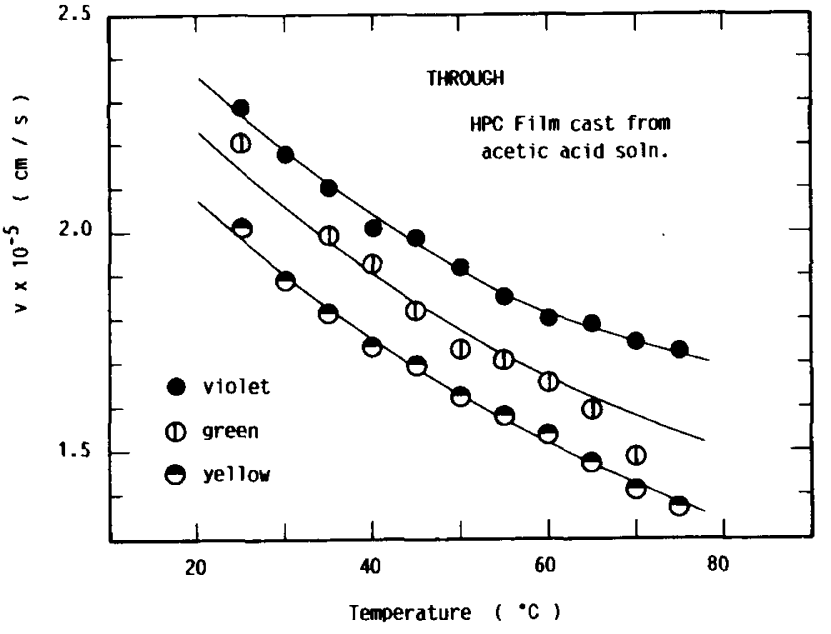

Figure 4. Temperature dependence of ultrasonic velocity in the direction of perpendicular to film surface for HPC films cast from acetic acid solutions.

in attenuation is caused by loosening of intermolecular interaction, it may be deduced that packing of cholesteric layers affects the persistence of interactions between cholesteric domains with increasing temperature. Attenuation in the planner direction is sufficiently higher than that in the perpendicular direction. This is 


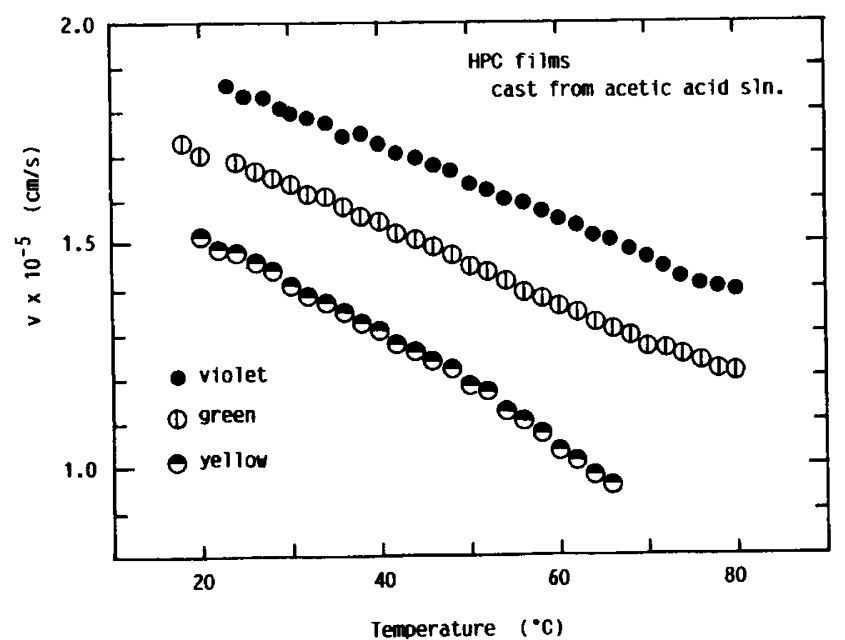

Figure 5. Temperature dependence of ultrasonic velocity in the direction of parallel to film surface for HPC films cast from acetic acid solutions.



Figure 6. Schematic representation of cholesteric structure. Arrows denote directions of ultrasonic waves.



Figure 7. Temperature dependence of ultrasonic attenuation coefficient in the direction of perpendicular to film surface for HPC films cast from acetic acid solutions.

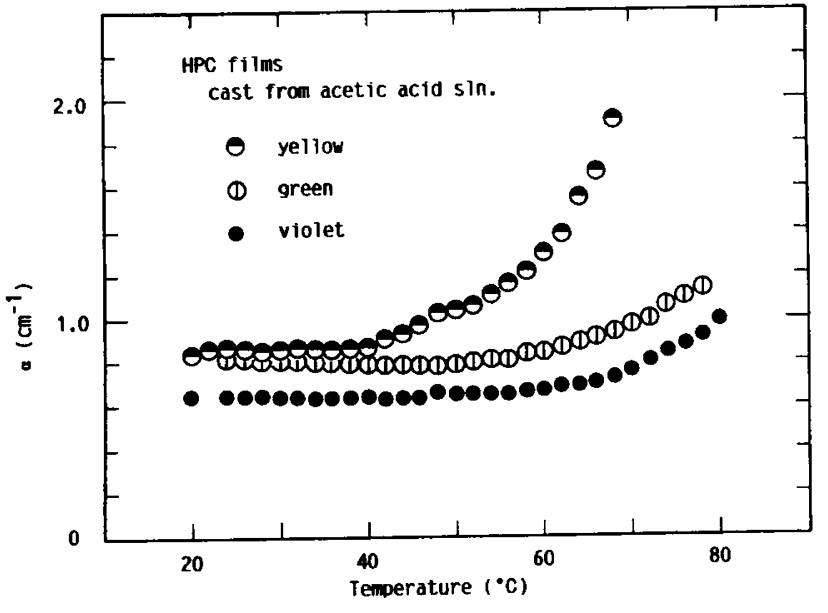

Figure 8. Temperature dependence of ultrasonic attenuation coefficient in the direction of parallel to film surface for HPC films cast from acetic acid solutions.

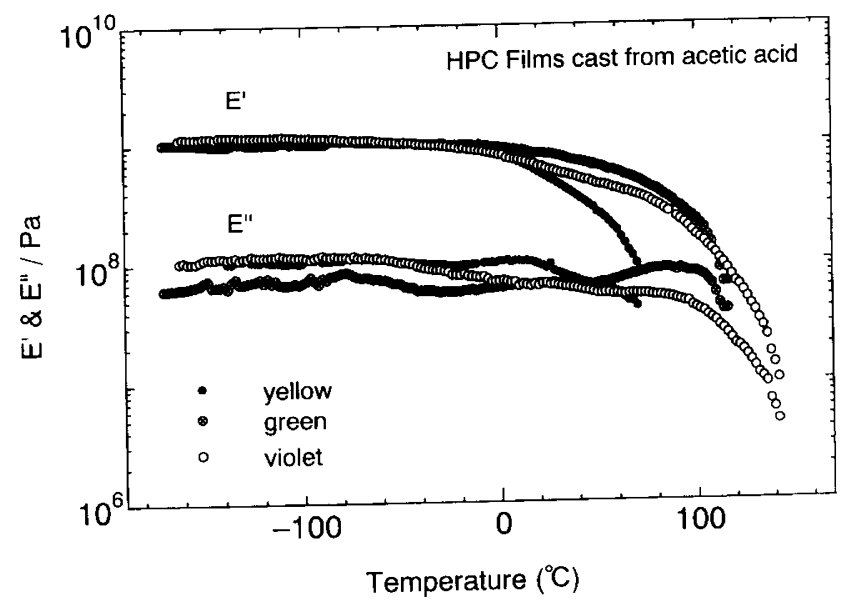

Figure 9. Temperature dependence of storage and loss moduli for HPC films cast from acetic acid solutions.

plausible since interactions in the perpendicular direction include looser interactions between cholesteric domains in the planner direction.

The cholesteric pitch dependence of ultrasonic properties of HPC-acetic acid films as described above was quite similar to that for other colored films prepared by casting from ethanol and dioxane.

Dynamic mechanical spectra measured at $110 \mathrm{~Hz}$ for HPC films cast from acetic acid are shown in Figures 9 and 10 . The broad maximum of loss tangent at about 25 ${ }^{\circ} \mathrm{C}$ measured in the direction parallel to the film is indicative of the glass transition. ${ }^{16,21}$ Above the glass transition temperature $T_{\mathrm{g}}, \tan \delta$ drastically increases and the storage modulus decreases smoothly. The onset of rise in $\tan \delta$ shifts to higher temperature with increasing cholesteric pitch of HPC films. The temperature for this onset is almost identical to the critical temperature for attenuation in the planner direction (see Figure 8). This suggests that drastic increase in $\tan \delta$ above $T_{\mathrm{g}}$ is attributed to increasing mobility of cholesteric domains, 


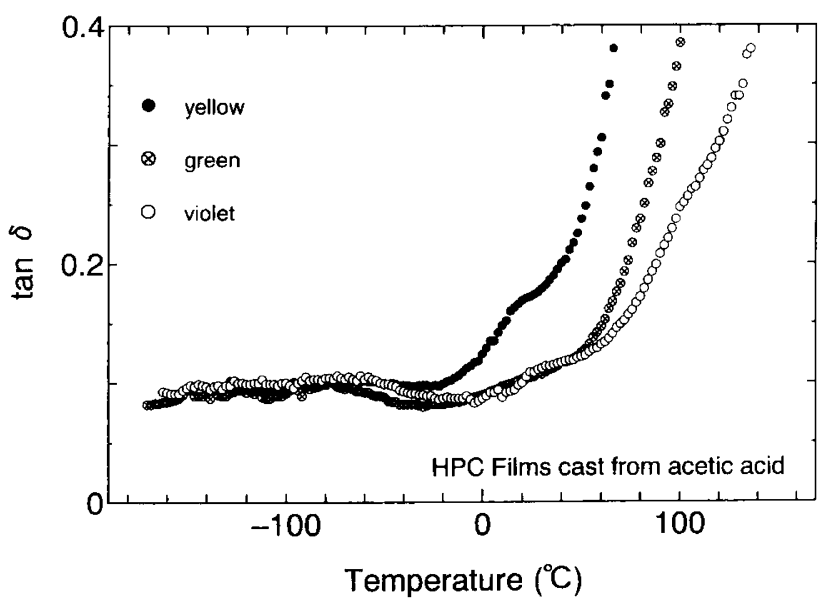

Figure 10. Temperature dependence of loss $\tan \delta$ for HPC films cast from acetic acid solutions.

due to release of inter-domain interaction.

\section{SUMMARY}

The molecular aggregation of hydroxypropyl-cellulose films prepared by casting from various solvents such as acetic acid, ethyl alcohol, dioxane, and water was examined by ultrasonic measurement in addition to dynamic mechanical measurement. For cholesteric colored HPC films, velocity and attenuation of ultrasonic waves in the planner and normal directions to the film surface were measured. Ultrasonic velocity and attenuation depended largely on cholesteric pitch. Anisotropy in ultrasonic properties was observed, indicating molecular aggregation or type of molecular interactions to differ for planner and normal directions of cholesteric layers.

\section{REFERENCES}

1. D. G. Gray, J. Appl. Polym. Sci., Appl. Polym. Symp., 37, 179 (1983).

2. R. D. Gilbert and P. A. Patton, Prog. Polym. Sci., 9, 115 (1983).

3. D. G. Gray, Faraday Discuss. Chem. Soc., 79, 257 (1985).

4. R.S. Werbowyj and D. G. Gray, Macromolecules, 13, 69 (1980).

5. J. Behda, J. F. Fellers, and J. L. White, Colloid Polym. Sci., 258, 1335 (1980).

6. T. Tsutsui and R. Tanaka, Polym. J., 12, 473 (1980).

7. R. S. Werbowyj and D. G. Gray, Macromolecules, 17, 1512 (1984).

8. A. Elliot and E. J. Ambrose, Faraday Discuss. Chem. Sci., 9, 246 (1950)

9. L. Onsager, Ann N. Y. Acad. Sci., 51, 627 (1947).

10. P. Flory, Proc. Roy. Soc. London -A, 234, 73 (1956).

11. C. Robinson, Trans. Faraday Soc., 52, 571 (1956).

12. C. Robinson, Mol. Cryst., 1, 467 (1966).

13. Hl. de Vries, Acta Cryst., 4, 219 (1951).

14. A. Tanaka, Y. Hamada, H. Onoda, T. Yamaguchi, and K. Nitta, Polym. J., 26, 599 (1994).

15. M. Horio, E. Kamei, and Y. Uchimura, J. Rheol, 13, 25 (1985).

16. J. S. Aspler and D. G. Gray, Polymer, 23, 43 (1982).

17. K. Shimamura, J. L. White, and J. F. Fellers, Appl. Polym. Sci., 26, 2165 (1981).

18. S. Suto, J. L. White, and J. F. Fellers, Rheol. Acta., 21, 62 (1982).

19. I. Uematsu and Y. Uematsu, "Advances in Polymer Science", Springer-Verlag, Berlin, 1984, pp 37-73.

20. S. N. Bhadani and D. G. Gray, Mol. Cryst. Liq. Cryst., 102, 255 (1984)

21. G. Charlet and D. G. Gray, Macromolecules, 20,33 (1987).

22. R. J. Samuels, J. Polym. Sci., A-2, 7, 1197 (1969). 